\title{
Cosmic Ray Origin and Future Travels with Velocities of CR Particles in Heliosphere, Galaxy and Universe
}

\author{
Lev Dorman ${ }^{1,2}$ * \\ ${ }^{I}$ Pushkov Institute of Terrestrial Magnetism, Ionosphere and Radiowave Propagation of the Russian \\ Academy of Sciences (IZMIRAN), Troitsk, Moscow, Russia \\ ${ }^{2}$ Israel Cosmic Ray and Space Weather Center with Emilio Segre Observatory on Mt. Hermon, affiliated \\ to Tel Aviv University, Ariel University, Shamir Research Institute, and Israel Space Agency, Israel
}

*Presenter author, e-mail: lid010529@gmail.com

\begin{abstract}
We will show that Solar Energetic Particles (SEP), as well as energetic particles generated in magnetospheres of the Earth, Jupiter, Saturn and other planets, in interplanetary space, and in atmospheres of stars have the same nature as Galactic and Intergalactic CR: they are all runaway particles from the Maxwell-Boltzmann distribution of background plasma where they were generated. Energy of these run-away particles is much higher than average energy of background thermal particles. It is shown in this work that the energy of all these run-away particles have the same general nature: it is always transfer energy from the Macro-objects and Macro-processes directly to Micro World (to charged runaway particles). This transfer energy is formatted in dynamic plasma with frozen in magnetic fields: really magnetic fields 'glues' billions thermal background particles into Macro-objects and Macroprocesses. So, thank to frozen in magnetic fields runaway particles can interact not only with thermal background particles (and loose energy), but also directly with Macro-objects and Macro-processes with very high macro-energy (many order higher than energy of run-away particle). Thermodynamically Macro-objects have much bigger "effective temperature" than runaway particles and though the energy always transferred from Macro World to runaway particles of Micro World. We also consider the future travels of space probes with velocities of CR particles of different energy, how looked stars, planets, Galaxy and other objects in the Universe, what time need for comfort acceleration and what self-time and Earth's-time need these travels to different objects. It is also important to estimate what will be radiation hazards during real relativistic travels in future.
\end{abstract}

37th International Cosmic Ray Conference (ICRC 2021) July 12th-23rd, 2021

Online - Berlin, Germany 


\section{The Matter of the Problem and Introduction}

When are talking on the origin of Cosmic Rays (or Astroparticles), usually have in view protons, nucleus of different $Z$, electrons, gamma-quants, and neutrino of high and very high energy generated in our Galaxy and in different astrophysical objects in the Universe, outside the Galaxy. Moreover, during many years energetic particles generated on the Sun were called as Solar Cosmic Rays (SCR, see Dorman and Miroshnichenko, 1968; Dorman, 1978; Miroshnichenko, 2001, 2015; Dorman and Dorman, 2014)), but now increased the tendency to rename this phenomenon to Solar Energetic Particles (SEP) event.

We will show that SEP, energetic particles generated in magnetospheres of the Earth, Jupiter, Saturn and other planets, in interplanetary space, and in atmospheres of stars have the same nature as Galactic and Intergalactic CR: they are all runaway particles from the Maxwell-Boltzmann distribution of background plasma where they were generated.

Energy of these run-away particles is much higher than average energy of background thermal particles. We will show in our report that the energy of all these run-away particles has the same general nature: it is always transfer energy from the Macro-objects and Macro-processes directly to Micro-objects (to charged runaway particles). This transfer energy is formatted in dynamic plasma with frozen in magnetic fields: really magnetic fields 'glues' thermal background particles in Macro-objects and Macroprocesses involved billions thermal particles. Through magnetic fields runaway particles interact not only with thermal background particles (and loose energy), but also directly interact with Macro-objects and Macro-processes with very high macro-energy (many order higher than energy of most energetic runaway particles) with energy transfer from Macro-world to Micro-world with energy increasing of runaway particles.

We come to conclusion that the main cause of origin of all types of Cosmic Rays in any objects and processes in the Universe is the transform energy from Macro-world directly to Micro-world through frozen in magnetic fields in plasmas. Let us outline that without frozen in magnetic fields run-away particles cannot interact with whole macro objects or macro-processes, but only with objects of Microworld (ions, electrons, atoms, molecules, and so on, and mostly with loosing energy). At the stage when in early Universe were formatted exited small density plasmas objects with frozen in magnetic fields, where formatted also Cosmic Rays of different types. Formatting energy spectrum of different types of $\mathrm{CR}$ is determined by the particle rate acceleration and loosing particles with increasing energy by increasing flux going out from region of acceleration; this phenomenon determined the maximum energy in spectrum (Dorman, 1957,1963a,b, 1972; Ginzburg and Syrovatsky, 1964; Berezhko et al., 1988; Berezinsky et al., 1990; Dorman, 2006, 2017a,b; Dorman and Dorman, 2014).

\section{Internal and external CR; multiple origin of CR}

There are internal CR, generated inside the background plasma of object considered, and external $\mathrm{CR}$ generated in other objects and propagated to the considered object. There are CR of different origin: Extragalactic or Metagalactic CR (ECR), Galactic CR (GCR), different types of Stellar CR (StCR), Solar CR (SCR), Heliospheric CR (HCR), Interplanetary CR (ICR), Magnetospheric CR (MCR), and so on. I think that in near future will be developed methods to investigate properties of CR separately in different astrophysical objects.

\subsection{Extragalactic or Metagalactic CR (ECR)}

ECR of very high energy (up to $10^{21} \mathrm{eV}$ or even up to $10^{22} \mathrm{eV}$ ) are generated in radio-galaxies, quasars and other powerful objects in the Universe and come through intergalactic space to our Galaxy, to the Heliosphere and into the Earth's atmosphere. Therefore, they are internal for Metagalaxy and external relative to our Galaxy and our Heliosphere.

\subsection{Galactic CR (GCR)}

GCR, with energy at least up to $10^{15}-10^{16} \mathrm{eV}$ (may be up to $10^{17} \mathrm{eV}$ ), are generated mainly by shock waves in supernova explosions and supernova remnants, in magnetospheres of pulsars and double stars, reaccelerated by shock waves in interstellar space and other possible objects in the Galaxy. These CR are internal relative to our Galaxy and external to our Heliosphere and the Earth's magnetosphere.

\subsection{Solar CR (SCR)}

$\mathrm{SCR}$, with energy up to $15-30 \mathrm{GeV}$, generated in the solar corona in periods of powerful solar flares, are 
internal CR for the Sun's corona and external for interplanetary space and the Earth's magnetosphere. SCR play important role in Heliosphere, and Earth's magnetosphere/atmosphere, but they role in general balance of GCR in the Galaxy (together with other stars) is expected negligible.

\subsection{Different types of Stellar CR (StCR)}

Stellar flares and shock waves in stellar winds are powerful sources for generation StCR with upper energy much bigger than Solar CR (may be up to $10^{11}-10^{12} \mathrm{eV}$ ). The role of StCR is important in the region not far from the corresponding star, but in general balance of GCR in the Galaxy the summary role of all StCR is expected negligible.

\subsection{Heliospheric CR (HCR)}

Heliospheric CR (HCR), with energy up to $\sim 100 \mathrm{MeV}$, are generated by the terminal shock at the boundary of the Heliosphere (when this particles were discovered at about 1960s, they was called as Anomaly CR or ACR, see Dorman, 1972, 2006). HCR are internal to our Heliosphere and external to the Earth's magnetosphere.

\subsection{Interplanetary CR (ICR)}

Interplanetary CR (ICR) also with energy up to $\sim 100 \mathrm{MeV}$ are generated by CME (Coronal Mass Ejections) and by powerful interplanetary shock waves from solar flares and CME. They are internal to our Heliosphere and external to the Earth's magnetosphere.

\subsection{Magnetospheric CR (MCR)}

Magnetospheric CR (MCR), with energy up to 10-20 MeV are generated in magnetospheres of Jupiter and Saturn, and up to 1-2 MeV in the magnetosphere of the Earth. MCR are generated inside the magnetospheres of all rotating magnetized planets interacted with solar or stellar winds. MCR are internal $\mathrm{CR}$ in the corresponding magnetosphere and external relative to Heliosphere and Stellar-spheres.

\section{Two different particle energy distributions in magnetized space plasma for background particles and runaway particles (Cosmic Ray particles)}

Let us consider the particle energy distribution in any magnetized dynamic space plasma. We can see that there are always two types of distributions, with a great differences in types of spectrums, in average energies and in density (several and many orders of magnitude). Let us consider, for example, Galactic Cosmic Rays in the Galaxy. The energy distribution of background particles in the Galaxy is $D_{B}\left(E_{k}\right) \sim E_{k} \exp \left(-E_{k} / k T\right)$ with average energy $\left\langle E_{k}\right\rangle_{B}=(3 / 2) k T \approx 1 \mathrm{eV}$ and density $\rho_{B} \sim 1 \mathrm{~cm}^{-3}$ with energy density $\left\langle E_{k}\right\rangle_{B} \times \rho_{B} \sim 1 \mathrm{eV} . \mathrm{cm}^{-3}$. The energy spectrum of GCR in our Galaxy is $D_{G C R} \sim E_{k}^{-\chi\left(E_{k}\right)}$ with average energy $\left\langle E_{k}\right\rangle_{G C R} \sim 10^{10} \mathrm{eV}$ and density $\rho_{G C R} \sim 10^{-10} \mathrm{~cm}^{-3}$ with energy density $\left\langle E_{k}\right\rangle_{G C R} \times \rho_{G C R} \sim 1 \mathrm{eV} . \mathrm{cm}^{-3}$. The same types of background particle energy distributions and runaway (Cosmic Ray) particle energy distributions we see in about all cases described in Section 2 . Let us note that when the energy density of any type of CR became bigger then energy density of background particles important role start to play nonlinear effects of CR: pressure of runaway particles and kinetic stream instabilities, which influenced on the structure and behavior of background plasma and finally lids to increasing of background particles energy density (may be it is the main cause why in many astrophysical objects CR energy density and background particles energy density are about equal, see in more details in Beresinsky et al., 1990; Dorman, , 2006, Chapter 3)

\section{Two Worlds in the Nature and runaway particles (CR particles, Astroparticles)}

The Nature in the present stage of the Universe consists from two Worlds with very different physical laws of their behavior.

1. Micro World: molecules, atoms, electrons, ions, photons, elementary particles, quarks and gluons, runaway particles (CR particles, Astroparticles). Micro World is ruled by Quantum Physics and Special Theory of Relativity. Micro World contains following different types of energy: a) Coupling energy between atoms and molecules which can be using during changing of aggregate state and in different chemical reactions ( $\sim \mathrm{eV}$ per one atom or per one molecule). This type of energy starts to use by people many thousand years ago (burning for obtain heat and preparing foods, metallic and other articles) and from about two hundred years ago up to present, for producing electro-energy, for moving cars, trains, sea ships, airplanes, rockets, space probes and so on). b) Coupling energy of protons and neutrons in nuclear of heavy atoms as $\mathrm{U}, \mathrm{Pu}$ and others ( $\sim$ tens $\mathrm{MeV}$ per atom). This type of energy starts to use by people 
only at the middle of 20-th century in the first for military aims, but then for obtaining electro-energy by Atomic Electro-Stations in many countries. c) Coupling energy of protons and neutrons in light nuclease as hydrogen, deuterium, and treaty during thermo-nuclear reactions ( $\sim$ several MeV per nucleus). This type of reactions $4 \mathrm{H} \rightarrow \mathrm{He}$ realized inside the Sun and many other stars, but people started to use it only at the middle of 20-th century in the first for military aims, but then was organized a great International research for obtaining electro-energy for people on the basis of thermo-nuclear reactions. d) Runaway particles (CR particles of many different types, Astroparticles). This part of Micro World contains total energy which is very small in comparison with other parts of Micro World, but some small part of runaway particles can reach very high energies up to $10^{21}-10^{22} \mathrm{eV}$, which until now are much bigger than can be reached on modern accelerators on the Earth.

2. Macro World with objects and processes in which involved billions objects of Micro World: Hard bodies, Liquids, Neutral Gases, Ionized Gases, Plasmas without and with frozen in magnetic fields, dark matter and dark energy, the Earth with Atmosphere and Ionosphere/Magnetosphere, other planets of Solar system, the Sun and stars, galaxies, quasars, black holes, dynamical Universe, and many other astrophysical objects. The Macro World is ruled by Classic Physics and General Theory of Relativity (if not consider the Big Bang and very short period after). On the Earth, the energy of Macro World start to use by people many thousand years ago and continue to use at present (e.g., kinetic energy of wind and rivers). It is interesting and important that runaway particles (CR particles of different types, Astroparticles) are accelerated by the objects and processes of Macro World (in plasmas with frozen in magnetic fields).

\section{Interactions of Micro World objects with objects of Macro World}

\subsection{On the possibility of direct interaction of Micro World objects (run-away particles) with objects of Macro World in case of hard bodies}

In this case, atoms and molecules of hard body have so small coupling energy $(\leq 1 \mathrm{eV})$ in comparison with energy of run-away particle, that they can be considered as free atoms and molecules. It means that run-away particle really interact only with objects of Micro World and loose energy. So, in this case it is only direct interaction of Micro World (run-away particles) with Micro World (atoms and molecules), but not direct interaction of Micro World (run-away particles) with Macro World.

5.2. On the possibility of direct interaction of Micro World objects (run-away particles) with objects of Macro World in cases of liquids, gases (including ionized gases but without frozen in magnetic field)

In all these cases runaway particles will be also interact directly only with atoms, molecules, electrons and ions (and loose energy), i.e. it will be direct interactions between Micro World's objects, but not Micro World objects with Macro World objects.

\subsection{On the possibility of direct interaction of Micro World objects (run-away particles) with objects of Macro World in case of ionized gases and space plasmas with frozen in magnetic field}

This case is very different from considered in Sections 5.1 and 5.2 because frozen in magnetic field formatted from billions of Micro-World objects the Macro-World object and processes, directly interacted with run-away particles (cosmic ray particles). By other words, in this case, as it was mention in Section 1, run-away particle will interact not only with Micro-World's objects (and mostly loose the energy), but also directly with objects and processes of Macro World with possibility of additional acceleration by taking some small part of energy from Macro World object and processes, formatted by frozen in magnetic field.

\subsection{Comparison of CR particles energy (Micro World) with energy of Macro World's objects directly interacted with CR particles through frozen in magnetic fields}

Let us, for example, consider CME (Coronal Mass Ejection) or shock wave (generated by CME) and their interactions with run-away particles. Let us consider CME near the Earth's orbit with following 
dimension, velocity, density, and total kinetic energy: $\mathrm{L} \approx 10^{12} \mathrm{~cm} ; \mathrm{u} \approx 500 \mathrm{~km} / \mathrm{s} \approx 5 \times 10^{7} \mathrm{~cm} / \mathrm{s} ; \rho \approx 5 \mathrm{~cm}^{-3}$; $\mathrm{W}_{\mathrm{k}} \approx \mathrm{L}^{3} \mathrm{\rho u}^{2} / 2 \approx 10^{28} \mathrm{erg} \approx 10^{40} \mathrm{eV}$. Kinetic energy of magnetic clouds in Galaxy has characteristics: $\mathrm{L} \approx$ $10^{18} \mathrm{~cm} ; \mathrm{u} \approx 10 \mathrm{~km} / \mathrm{s} \approx 5 \times 10^{6} \mathrm{~cm} / \mathrm{s} ; \rho \approx 1 \mathrm{~cm}^{-3} ; \mathrm{W}_{\mathrm{k}} \approx \mathrm{L}^{3} \rho \mathrm{u}^{2} / 2 \approx 10^{43} \mathrm{erg} \approx 10^{55} \mathrm{eV}$. The same situation we see not only in the interplanetary space and in the Galaxy, but also in Magnetospheres of planets interacted with solar and stellar winds, on the Sun and many types of stars, during Supernova explosions and in Supernova remnants, on many powerful objects in Metagalaxy: the energy of Macro-Worlds objects and processes, interacted with local runaway particles (CR particles, Astroparticles), is many orders higher than the maximum of local CR particles energy. It means that CR phenomenon is very far from thermo-dynamical equilibrium with Macro-Worlds objects and processes. It means that energy of Macro-Worlds objects and processes in plasmas with frozen in magnetic fields by different mechanisms continuously transferred to runaway particles (Cosmic Rays, Astroparticles).

\section{Mechanisms of runaway particles (CR, Astroparticles) generation: what is the main driver of charged particles acceleration?}

\subsection{Stochastic acceleration}

The mechanism of stochastic acceleration of particles by chaotically moving interstellar magnetic clouds has developed by Fermi (1949). According to E. Fermi, at each collision of a charged particle moving with velocity $\mathbf{v}$, with magnetic cloud moving with velocity $\mathbf{u}$, changes the energy of charged particle will be determined by the relation $(\Delta E / E)_{ \pm}= \pm 2 u v / c^{2}$, where the upper sign is for head-on collisions and bottom sign for overtaking collisions. If $\lambda$ is the mean free path for particle collisions with magnetic clouds, the corresponding frequencies $\xi_{ \pm}$correspondingly for head-on and overtaking collisions will be $\xi_{ \pm}=(v \pm u) / 2 \lambda$. The average change energy per unit of time will be $d E / d t=(\Delta E)_{+} \xi_{+}+(\Delta E)_{-} \xi_{-}=\alpha E$, where $\alpha=2 u^{2} v / \lambda c^{2}$ is the so called parameter of acceleration.

Let us note that more detail consideration (Dorman, 1983; see in Dorman, M2006, Section 4.3 of Chapter 4) shows that taking into account small change radius of curvature during moving inside frozen in magnetic field, the increasing and decreasing energy during head-on and overtaking collisions, correspondingly, will be $(\Delta E / E)_{ \pm}= \pm 2 u v / c^{2}+2 u^{2} / c^{2}$ and in this case $\alpha=4 u^{2} v / \lambda c^{2}$, i.e., the stochastic mechanism really is two times more effective than was obtained originally in Fermi (1949).

Fermi's mechanism of stochastic acceleration of charged particles was the first physically clearly mechanism, which in subsequent years was developed and applied to the problem of CR acceleration and reacceleration in many papers (e.g., Seo and Ptuskin, 1994; Michałek et al., 1999; see review in Dorman, M2006, Chapter 4). In this mechanism is clearly seen that acceleration of runaway particles (CR, Astroparticles) realized by transferring kinetic energy of macro objects to energy of micro particles through frozen in magnetic field.

\subsection{Drift acceleration by shock waves}

If the shock wave propagated in plasma with frozen in magnetic field, charged particles near the shock front will be drift along electric field and accelerated (drift acceleration). This mechanism was

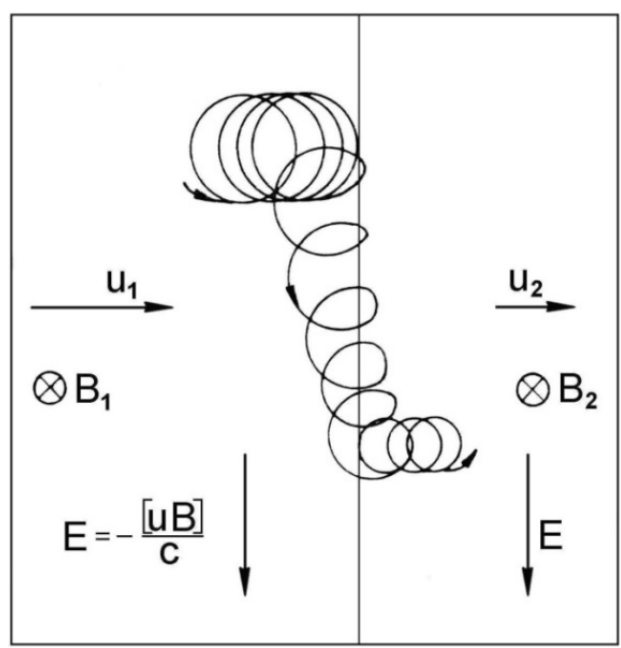
supposed by Dorman and Freidman (1959) for explaining effect of galactic CR pre-increase before the start of big magnetic storms with sudden commencement (caused by CME and interplanetary shock waves from the Sun, see Figure 1.

Figure 1. Charged particle trajectory in shock wave (coordinate system related to the wave front). According to Dorman and Freidman (1959).

In this case $\mathrm{u}_{1} \mathrm{H}_{1}=\mathrm{u}_{2} \mathrm{H}_{2}$ and electric field $\mathrm{E}_{1}=\mathrm{E}_{2}$; the increasing energy $\mathrm{E}$ of charged accelerating particle will be $\Delta \mathrm{E}=\mathrm{ZeE}_{1} \mathrm{~L}=\mathrm{ZeE}_{2} \mathrm{~L}$, where $\mathrm{Ze}$ is the charge of accelerated particle and $\mathrm{L}$ - the way of drift along electric field on the front of shock wave. Later this mechanism was developed in details by many authorssee review in Dorman, M2006, Chapter 4. In this case 
we also see that the main driving of charged particle acceleration is the transfer energy to particle through frozen in magnetic field from kinetic energy of shock wave, i.e. again, direct transfer energy from macroobjects and macro-processes to the micro-objects (runaway particles, CR, Astroparticles).

\subsection{Regular or diffusive shock wave acceleration}

The regular or diffusive acceleration of charged particles during multiple interaction with shock wave were supposed and developed by Krymsky (1977), Axford et al. (1977), Bell (1978a,b). This type of particle acceleration by shock wave is called as regular shock acceleration (because at each crossing of shock front particle gain the energy) or diffusive shock acceleration (because the multi-crossing of shock front is caused by particle scattering and diffusion through magnetic inhomogeneities behind and before the shock front).

It is important that as it was shown by Krymsky (1977), the expected spectrum of accelerated particles will be power type: $\propto p^{-\gamma} ; \gamma=\frac{u_{1}+2 u_{2}}{u_{1}-u_{2}}=\frac{\sigma+2}{\sigma-1}$, where $p$ is the momentum of accelerated particles and $\sigma=u_{1} / u_{2}=\rho_{2} / \rho_{1}=H_{2} / H_{1}$ is the degree of shock compression ratio by the shock wave. It is important to note that with increasing $\sigma$ from 2 (weak shock wave) to 4 (strong shock wave) the value of $\gamma$ decreases from 4 to 2 in agreement with what is observed for galactic CR (it means that main source of galactic CR are strong shock waves). This model was then developed in details (especially in applications to the problem of CR acceleration by shock waves in Supernova remnants, in the interplanetary space by shock waves driving by coronal mass ejections, by bow shocks in the planetary magnetospheres, by the terminal shock wave in the Heliosphere, and other types of shock waves) by many authors; see reviews in Berezhko et al. (M1988), Berezinsky et al. (M1990), Moskalenko (2003), Dorman (M2006). Let us outline that the main properties of the observed nonthermal emission of Supernova remnants are consistent with the efficient CR acceleration at the Supernova blast wave. The existing data together with the development of nonlinear kinetic theory of CR acceleration in Supernova remnants provide the evidence that galactic Supernova remnants are the main source of galactic CR up to the energy $10^{15} \mathrm{eV}-$ $10^{16} \mathrm{eV}$. We also see that in all described cases of diffuse shock wave acceleration the main driver of runaway particles (CR, Astroparticles) generation is direct energy transfer kinetic energy of shock waves through frozen in magnetic field (objects and processes of Macro World) directly to charged energetic particles, CR, Astroparticles (objects of Micro World).

\subsection{Charged energetic particle generation in reconnection processes}

In many different reconnection processes of space magnetized plasma we also see that during annihilation magnetic field is generated electric field which accelerates about all runaway charged particles (Cosmic Rays, Astroparticles) in the volume of reconnection (Sweet, 1958, 1969; Parker, 1963; Petchek, 1964; Syrovatsky, 1966, 1967, 1968, 1971, 1981; Hesse et al., 2005; Zelenyi et al., 2010; Liu et al., 2017b. Artemyev et al., 2019). After escaping from acceleration region runaway charged particles (Cosmic Rays, Astroparticles) interacted with matter and fields in surrounded space plasma with changing both initial composition and energy spectrum. In this case the main driver of generation runaway charged particles (Cosmic Rays, Astroparticles) again is the direct transfer energy of Macro World object and process (reconnection phenomenon) directly to Micro World objects thanks to frozen in magnetic field.

\section{Formation of composition and energy spectrum of runaway charged particles (Cosmic Rays, Astroparticles) and possible maximum energy}

The following processes are important for formation of composition and energy spectrum of runaway charged particles (Cosmic Rays, Astroparticles) and possible maximum energy:

1. How changed the rate of energy increase during the acceleration process with increasing particle energy, ionization and other losses inside the source, nuclear interactions and change the composition during acceleration.

2. Escaping of runaway charged particles (Cosmic Rays, Astroparticles) from the source, dependence of energy, maximum energy of particle trapping. As rule, the probability of escaping increased with increasing the particle energy; this phenomenon influenced on the upper limit of runaway particles (Cosmic Rays, Astroparticles) energy spectrum.

3. Reacceleration of runaway charged particles (Cosmic Rays, Astroparticles) outside the sources and energy losing on ionization and nuclear interactions (important for small and middle energy), on interactions with magnetic field (synchrotron radiation; important for electrons), losing energy on 
interactions with photons (especially with relict photons at $2.7^{\circ} \mathrm{K}$ ) important for very high energy particles with $\mathrm{E}>10^{18}-10^{20} \mathrm{eV}$, generated in the Universe on great distances from our Galaxy.

These problems are considered in details in monographs of Ginzburg and Syrovatsky (1964), Dorman (1972), Berezhko et al. (1988), Berezinsky et al. (1990), Schlickeiser (2001), Dorman (2006), and in many papers.

\section{Future Travels on Relativistic Space-Probes}

By help of controlled thermo-nuclear fusion can be created powerful ion engines for relativistic space probes. In this case will be real to attain velocities as CR particles. Let us consider observed maximum $\mathrm{CR}$ proton energy about $10^{19} \mathrm{eV}$. In the self-system coordinate it will transfer our Galaxy with dimension about $10^{5}$ light years for 5 minutes, stars and planets will be compressed in the direction of moving in $10^{10}$ times, but in perpendicular direction the dimensions will be not change. For protons $10 \mathrm{GeV}$ the self-time for transfer our Galaxy will be $10^{4}$ light years; stars and planets will be compressed in the direction of moving in 10 times (they will be rotated 10 times faster).

\section{Conclusion}

The main cause of origin of all types of runaway charged energetic particles (Cosmic Rays, Astroparticles) in different objects and processes in the Universe is the transform energy from Macroworld directly to Micro-world through frozen in magnetic fields in plasmas. Without frozen in magnetic fields runaway particles (Cosmic Rays, Astroparticles) cannot interact with whole macro objects or macro-processes, but only with objects of Micro-world (ions, electrons, atoms, molecules, and so on), and mostly with loosing energy. Of course, the energy of runaway particles (Cosmic Rays, Astroparticles) finally will go back to Macro-world through ionization and other energy loosing processes, and through nonlinear effects (CR pressure and kinetic stream instabilities), but it can happen during long time after escaping from sources (e.g., for solar and magnetospheric CR - hours and days, but for galactic CR about $3 \times 10^{7}$ years, and for very high energy metagalactic $\mathrm{CR}-$ much more than $10^{8}$ years).

\section{References}

Axford W.I., E. Leer, and G. Skadron, 1977. "The acceleration of cosmic rays by shock waves", Proc. 15th Intern. Cosmic Rays Conf., Plovdiv, 11, 132-137.

Bell A.R., 1978a. "The acceleration of cosmic ray in shock fronts. I.", Monthly Notices of the Royal Astronomical Society, 182, No.1, 147-156.

Bell A.R., 1978b. "The acceleration of cosmic rays in shock fronts. II", Monthly Notices of the Royal Astronomical Society, 182, No.2, 443-455.

Berezhko E.G., V.K. Elshin, G.F. Krymsky, and G.F. Petukhov, 1988. Cosmic Ray Generation by Shock Waves, Nauka, Novosibirsk (in Russian).

Berezinsky V.S., S.V. Bulanov, V.L. Ginzburg, V.A. Dogiel, and V.S. Ptuskin (ed. V.L. Ginzburg), M1990. Astrophysics of Cosmic Rays, Physmatgiz, Moscow (in Russian). English translation: NorthHolland, Amsterdam, M1990.

Dorman L.I., 1959. "On the beginning stage of charge particles acceleration", Proc. 6th Intern. Cosmic Ray Conf., Moscow, 3, 245-252.

Dorman, L.I., 1972. Acceleration Processes in Space. VINITI, Moscow, in Russian.

Dorman Lev I., 1978. Cosmic Rays of Solar Origin, VINITI, Moscow (in series "Summary of Science", Space Investigations, Vol.12), in Russian.

Dorman L.I., 1983. "On the formation of energy spectrum and character of particle energy gain in the statistical mechanism of acceleration". Cosmic Rays, Moscow, NAUKA, Vol. 23, pp. 5-13.

Dorman L.I., 2006. Cosmic Ray Interactions, Propagation, and Acceleration in Space Plasmas. Springer, Dordrecht/Boston/London/Heidelberg.

Dorman L.I. and I.V. Dorman, 2014. Cosmic Ray History, Nova Science Publishers, New York.

Dorman L.I. and G.I. Freidman, 1959. "On the possibility of charged particle acceleration by shock waves in magnetized plasma", Problems of Magnetic Hydrodynamics and Plasma Dynamics, Vol. 3, Ac. Sci. Lat. SSR, Riga, 77-81.

Dorman L.I. and L.I. Miroshnichenko, 1968. Solar Cosmic Rays. FIZMATGIZ, Moscow, pp. 508, in Russian. English translation published for NASA and NSF in Delhi, M1976.

Fermi E., 1949. "On the Origin of the Cosmic Radiation”, Phys. Rev., 75 (8), 1169-1174.

Ginzburg V.L. and S.I. Syrovatsky, 1964. The Origin of Cosmic Rays, Publ. Acad. Sci. USSR, Moscow 
(in Russian). In English: Pergamon Press, 1964.

Krymsky G.F., 1977. "Regular mechanism of charged, particle acceleration at shock front", Dokl. Akad. Nauk. SSSR, 234, No. 6, 1306-1308. Translation from Russian to English: Sov. Phys. Doklady, 22, 327-328 (1977).

Lagage P.O. and C.J. Cesarsky, 1983. "The maximum energy of cosmic rays accelerated by supernova shocks", Astron. and Astrophys., 125, No. 2, 249-257.

Malkov M.A. and L.O'C. Drury, 2001. "Nonlinear theory of diffusive acceleration of particles by shock waves", Rep. Progr. Phys., 64, No. 4, 429-481.

Michałek G., M. Ostrowski, and G. Siemieniec-Oziębło, 1999. "Stochastic Fermi acceleration in turbulent fields with non-vanishing wave helicities", Proc. 26th Intern. Cosmic Ray Conf., Salt Lake City, 6, 284-287.

Miroshnichenko L.I., 2001. Solar Cosmic Rays, Kluwer Ac. Publishers, Dordrecht.

Miroshnichenko L.I., 2015. Solar Cosmic Rays - Fundamentals and Applications, Second Edition, Astrophysics and Space Science Library, Volume 405, Springer International Publishing. Heidelberg.

Moskalenko I.V., 2003. "Cosmic Ray Propagation and Acceleration", Proc. 28-th Intern. Cosmic Ray Conf., Tsukuba, 8, 183-204.

Parker E.N., 1963. "The solar-flare phenomenon and the theory of reconnection and annihilation of magnetic fields", Astrophys. J. Suppl., 8, 177-211.

Petchek H.E., 1964. "Magnetic field annihilation", In Proc. AAS-NASA Sympos. on Physics of Solar Flares, NASA SP-50, Ed. by W.N. Hess, Washington, 425-439.

Ptuskin V.S. and V.N. Zirakashvili, 2003. "Limits on diffusive shock acceleration in supernova remnants in the presence of cosmic-ray streaming instability and wave dissipation", Astronomy and Astrophysics, 403, No. 1, 1-10.

Ptuskin V., V. Zirakashvili, and E.-S. Seo, 2010. "Spectrum of galactic cosmic rays accelerated in supernova remnants", Astrophys. J., 718, 31-36.

Reames D.V., L.M. Barbier, and C.K. Ng, 1996. "The spatial distribution of particles accelerated by coronal mass ejection-driven shocks", Astrophys. J., 466, No. 1, Part 1, 473-486.

Schatzman E., 1963. "On the acceleration of particles in shock fronts", Annales de Astrophysique, 26, No. 3, 234-249.

Schlickeiser R., 2001. Cosmic Ray Astrophysics. Springer, Berlin.

Seo E.S. and V.S. Ptuskin, 1994. "Stochastic reacceleration of cosmic rays in the interstellar medium", Astrophys. J., 431, 705-714.

Shabansky V.P., 1961. "Particle acceleration during passage of hydromagnetic shock front", Zh. Eksp. Teor. Fiz., 41, No.4 (10), 1107-1111.

Shabansky V.P., 1966. "Interaction of energetic charged particles with hydromagnetic waves", Geomagnetism and Aeronomy, 6, No.3, 472-478.

Sweet A., 1958. "The production of high energy particles in solar flares", Nuovo Cinento, 8, Suppl. 2, 188-196.

Sweet P.A., 1969. "Mechanisms of Solar Flares", Ann. Rev. Astron. Astrophys., 7, 149-176.

Syrovatsky S.I., 1966. "Dynamic dissipation of magnetic field and particle acceleration", Astronom. J., Moscow, 43, No.2, 340-355.

Syrovatsky S.I., 1967. "Fast particle generation in the space", Izv. Acad. of Science USSR, Ser. Phys., 31, No.8, 1303-1306.

Syrovatsky S.I., 1968. "Particle acceleration in the space", Proc. 5th All-Union Summer School of Cosmophysics, Apatity, 58-72.

Syrovatsky S.I., 1971. "Current sheets and particle acceleration in the space", Proc. Intern. Seminar on Cosmic Ray Generation on the Sun, Moscow, 15-36.

Syrovatsky S.I., 1981. "Pinch sheets and reconnection in astrophysics", Ann. Rev. Astron. Astrophys., 19, 163-229.

Vasilyev V.N., I.N. Toptygin, and A.A. Chirkov, 1978. "Interaction of high-energy particles with a shock front in turbulent medium", Geomagnetism and Aeronomy, 18, No.3, 415-422.

Velinov P.I.Y., 1971. On the Ionization Losses Influence on Cosmic Ray Spectrum. Geomagnetism and Aeronomy, 11, No. 3, 424-428.

Zelenyi L.M., A.V. Artemyev, Kh.V. Malova, A.A. Petrukovich, and R. Nakamura, 2010. "Metastability of current sheets", Physics - Uspekhi, 53 (9), 933-941. 